\title{
The Planck Constant Was Not a Universal Constant
}

\author{
Koshun Suto \\ Chudai-JI Temple, Isesaki, Japan \\ Email: koshun_suto129@mbr.nifty.com
}

How to cite this paper: Suto, K. (2020) The Planck Constant Was Not a Universal Constant. Journal of Applied Mathematics and Physics, 8, 456-463.

https://doi.org/10.4236/jamp.2020.83035

Received: January 31, 2020

Accepted: March 7, 2020

Published: March 10, 2020

Copyright $\odot 2020$ by author(s) and Scientific Research Publishing Inc. This work is licensed under the Creative Commons Attribution International License (CC BY 4.0).

http://creativecommons.org/licenses/by/4.0/

\begin{abstract}
According to traditional classical quantum theory, due to the prior existence of the Planck constant, considered a universal constant, it is thought that the energy of a photon can be determined if its frequency is known, and the wavelength of a quantum can be determined if its momentum is known ( $E=h v$ and $\lambda=h / p$ ). In this paper, however, the Planck constant only comes into existence when $m_{\mathrm{e}} c \lambda_{\mathrm{C}}$ is replaced with $h$. There is no problem with introducing $h$ to simplify equations, but quantum mechanics is not affected even if there is no symbol $h$. The physicists at the beginning of the 20th century overestimated the Planck constant, and this gave rise to universal constants that do not exist in the natural world in itself.
\end{abstract}

\section{Keywords}

Planck Constant, Universal Constants, Fundamental Physical Constants

\section{Introduction}

In 1900, when deriving a formula that derived an experimental value of black-body radiation, Planck proposed the quantum hypothesis stating that the energy of a harmonic oscillator with oscillation frequency $v$ would quantize at the integral multiple of $h v$. This was the first time that the Planck constant $h$ appeared in physics theory [1]. Since this time, the Planck constant has been thought to be a universal constant defined in the realm of quantum theory, but the essence of this constant cannot be considered to be well understood. In this paper, using non-historic reasoning, the true essence of this constant is revealed.

Beforehand, let us verify the following points regarding fundamental physical constants and the Planck constant. Fundamental physical constants play an es- 
sential part in elementary formulas that describe natural phenomena and can be largely divided into universal constants and material constants.

Universal constants include the speed of light in vacuum $c$, Newtonian constant of gravitation $G$, and Planck constant $h$.

Material constants can be divided into micro material constants and macro material constants. Also, micro material constants can be divided into atomic and nuclear constants and electromagnetic constants.

Atomic and nuclear constants include the electron rest mass $m_{\mathrm{e}}$, electron's Compton wavelength $\lambda_{\mathrm{C}}$, and include such constants as the fine-structure constant $\alpha$ and the Rydberg constant $R_{\infty}$. Electromagnetic constants include the elementary charge $e$.

The Boltzmann constant $k$ and the Avogadro constant $N_{\mathrm{A}}$ are examples of macro material constants.

However, the Planck constant $h$ is thought to be a universal constant representative of quantum mechanics and not dependent on human definition, unlike micro material constants such as $\alpha$ or $R_{\infty}$.

Because the Planck constant has an action quantity dimension, it was at first called an action quantum when quantum theory originally emerged. $h$ appears in the inequality $\Delta x \Delta p_{x} \geq \hbar / 2$ when Heisenberg discovered the uncertainty principle in 1927. Planck constant $h$, along with the speed of light in vacuum $c$ and the Newtonian constant of gravitation $G$, also plays an important role when assembling Planck units from universal constants. From the above, the Planck constant is a constant by name, but it has come to be strongly regarded as being the smallest unit of angular momentum.

Incidentally, in deriving the equation for the energy levels of the hydrogen atom, Bohr assumed the following quantum condition including the Planck constant:

$$
p_{n} \cdot 2 \pi r_{n}=2 \pi n \hbar, \quad n=1,2, \cdots
$$

Taking this quantum condition as a departure point, Bohr derived the following energy levels [2].

$$
E_{n}=-\frac{1}{2}\left(\frac{1}{4 \pi \varepsilon_{0}}\right)^{2} \frac{m_{\mathrm{e}} e^{4}}{\hbar^{2}} \cdot \frac{1}{n^{2}}=-\frac{\alpha^{2} m_{\mathrm{e}} c^{2}}{2 n^{2}}, \quad n=1,2, \cdots .
$$

Here, $\alpha$ is the following fine-structure constant.

$$
\alpha=\frac{e^{2}}{4 \pi \varepsilon_{0} \hbar c} .
$$

However, Equation (2) is a formula of classical quantum theory, and does not take into account the theory of relativity. When the theory of relativity is taken into account, the following energy levels can be derived [3].

$$
E_{\mathrm{re}, n}=m_{\mathrm{e}} \mathrm{c}^{2}\left[\left(\frac{n^{2}}{n^{2}+\alpha^{2}}\right)^{1 / 2}-1\right], \quad n=0,1,2, \cdots .
$$


The "re" in $E_{\mathrm{re}}$ stands for "relativistic".

Equation (4) is an elementary formula that includes only the principal quantum number $n$.

Here, let's examine the relationship between Equation (2) and Equation (4).

If the part in parentheses in Equation (4) is developed as a Taylor series,

$$
\begin{aligned}
E_{\mathrm{re}, n} & =m_{\mathrm{e}} c^{2}\left[\left(1-\frac{\alpha^{2}}{2 n^{2}}+\frac{3 \alpha^{4}}{8 n^{4}}-\frac{5 \alpha^{6}}{16 n^{6}}+\cdots\right)-1\right] \\
& \approx-\frac{\alpha^{2} m_{\mathrm{e}} c^{2}}{2 n^{2}} .
\end{aligned}
$$

From this, it is evident that Equation (2) derived by Bohr is an approximation of Equation (4) [4].

Incidentally, the quantum condition is not assumed when deriving Equation (4). If the quantum condition is necessary, the following quantum condition can be inferred from the derived Equation (4) [5].

$$
p_{n} \cdot 2 \pi r_{n}=2 \pi \hbar \cdot \frac{n}{2}\left[1+\left(1+\frac{\alpha^{2}}{n^{2}}\right)^{1 / 2}\right] .
$$

However, it is difficult to predict and assume a complex condition like Equation (6) at the beginning. In Equation (6), the Planck constant doesn't seem to play an essential role for values of $\alpha$ or higher. Also, the Planck constant does not play an important role when deriving Equation (4).

The author has shown that the following quantization procedure is more important than quantum condition (1) [6].

$$
\frac{v_{n}}{c}=\frac{\alpha}{n} .
$$

Equation (7) is built into Equation (1). Equation (7) is a mathematical procedure for shifting from the classical world dominated by continuity to the discrete quantum world.

Here, let's multiply both sides of Equation (7) by the formula for classical kinetic energy $(1 / 2) m_{\mathrm{e}} v_{n}$. When this is done, we obtain:

$$
\frac{1}{2} m_{\mathrm{e}} v_{n} \cdot \frac{v_{n}}{c}=\frac{1}{2} m_{\mathrm{e}} v_{n} \cdot \frac{\alpha}{n} .
$$

If $v_{n}=\alpha c / n$ found from Equation (7) is used, then the energy levels (2) derived by Bohr are obtained. That is,

$$
E_{n}=-\frac{1}{2} m_{\mathrm{e}} v_{n}^{2}=-\frac{1}{2} m_{\mathrm{e}} \frac{\alpha^{2} c^{2}}{n^{2}}=-\frac{\alpha^{2} m_{\mathrm{e}} c^{2}}{2 n^{2}} .
$$

Equation (4) can be derived from Equation (7) [7], but Equation (4) cannot be derived from Equation (1).

Incidentally, the following Rydberg formula is available for calculating spectrum wavelength.

$$
\frac{1}{\lambda}=R_{\infty}\left(\frac{1}{m^{2}}-\frac{1}{n^{2}}\right), \quad m=1,2, \cdots, \quad n=m+1, m+2, \cdots .
$$


Recently, the author has shown that Equation (10) is correct within the scope of classical theory, but it becomes an approximation when the theory of relativity is taken into account [8]. In this way, it has been shown that even the Rydberg constant $R_{\infty}$ in Equation (10) cannot, strictly speaking, be regarded as a fundamental physical constant.

It has also been pointed out that there are ultra-low energy levels that cannot be predicted by quantum mechanics in the hydrogen atom. The incompleteness of quantum mechanics has also become evident [9] [10] [11].

Thus, the next section examines whether Planck constant can truly be called a universal constant.

\section{Planck Constant Derived from Fundamental Physical Constant}

Below is Einstein's formula expressing the equality of energy and mass [12].

$$
E=m c^{2} \text {. }
$$

Meanwhile, Einstein's relational expression which applies Planck's quantum hypothesis to photons is as follows [13].

$$
E=h v .
$$

The photon's energy $E$ is proportional to its frequency $v$, and this constant of proportionality is known as the Planck constant. Equation (11) and Equation (12) are traditionally thought to be representative equation of the special theory of relativity and quantum mechanics, the roots of modern physics, and these two equations have been thought to have similar importance.

Now, if $m_{\mathrm{e}}$ is the mass of an electron, an electron's rest mass energy $E_{0}$ can be represented by the following equation.

$$
E_{0}=m_{\mathrm{e}} c^{2} .
$$

If the law of conservation of momentum is taken into account, then a single photon of energy $m_{\mathrm{e}} c^{2}$ is never emitted from an electron with rest mass energy $m_{\mathrm{e}} c^{2}$.

However, this does not mean that the existence of a photon with energy equal to $m_{\mathrm{e}} c^{2}$ is forbidden.

If $v_{0}$ is the frequency of a photon carrying an amount of energy equivalent to $E_{0}$, the following is true.

$$
E_{0}=h v_{0} .
$$

If we subsequently combine equals from Equation (13) and Equation (14), we obtain:

$$
m_{\mathrm{e}} c^{2}=h v_{0} .
$$

Here, the left half is the energy contained within the electron. This energy is emitted as multiple photons when it is externally emitted. The right half of this equation is the sum of all of this photon energy as represented by the frequency of a photon when converted into a single photon. Fundamentally these two types 
of energy have different characteristics, but from a quantitative perspective, it is possible to combine them as equals.

Incidentally, an electron's Compton wavelength $\lambda_{\mathrm{C}}$ is represented by the following equation.

$$
\lambda_{\mathrm{C}}=\frac{h}{m_{\mathrm{e}} c} .
$$

The Planck constant can be defined as follows from Equation (16).

$$
h=m_{\mathrm{e}} c \lambda_{\mathrm{C}} .
$$

Incidentally, Equation (13) can be written as follows.

$$
E_{0}=m_{\mathrm{e}} c \cdot \lambda_{0} v_{0} \text {. }
$$

$\lambda_{0}$ is the wavelength of a photon with energy $m_{\mathrm{e}} c^{2}$, but when Equation (17) is taken into account, Equation (18) can be written as follows.

$$
E_{0}=m_{\mathrm{e}} c \lambda_{\mathrm{C}} \nu_{0} .
$$

Comparing Equation (17) and Equation (18), it is evident that $\lambda_{0}$ in Equation (18) matches the electron's Compton wavelength $\lambda_{\mathrm{C}}$.

Thus, if we call $v_{0}$ the Compton frequency, and rewrite it as $v_{\mathrm{C}}$, then Equation (18) becomes as follows.

$$
E_{0}=m_{\mathrm{e}} c^{2}=m_{\mathrm{e}} c \lambda_{\mathrm{C}} v_{\mathrm{C}}=h v_{\mathrm{C}}
$$

Next, let's consider the case where the energy of the photon is not $m_{\mathrm{e}} c^{2}$ but rather $m c^{2}$.

The following relation holds between $m$ and $m_{\mathrm{e}}$. (Naturally, $m$ and $m_{\mathrm{e}}$ are not the mass of the photon.)

$$
E=m c^{2}=a m_{\mathrm{e}} c^{2}, \text { where } 0<a .
$$

At this time, the following relation holds.

$$
m c^{2}=a m_{\mathrm{e}} c^{2}=a m_{\mathrm{e}} c \lambda_{\mathrm{C}} v_{\mathrm{C}}=m_{\mathrm{e}} c \lambda_{\mathrm{C}} \cdot a v_{\mathrm{C}}=h v \text {, where } v=a v_{\mathrm{C}} .
$$

From Equation (21) and Equation (22) it is evident that all photon energies can be described using the following formula.

$$
E=h v \text {. }
$$

\section{Discussion}

We next substitute the following values for physical quantities in $m_{\mathrm{e}} c \lambda_{\mathrm{C}}$ [14].

$$
\begin{aligned}
& m_{\mathrm{e}}=9.1093837015 \times 10^{-31} \mathrm{~kg} . \\
& c=2.99792458 \times 10^{8} \mathrm{~m} \cdot \mathrm{s}^{-1} . \\
& \lambda_{\mathrm{C}}=2.42631023867 \times 10^{-12} \mathrm{~m} .
\end{aligned}
$$

By doing so, $m_{\mathrm{e}} c \lambda_{\mathrm{C}}$ becomes as follows.

$$
m_{\mathrm{e}} c \lambda_{\mathrm{C}}=6.62607015 \times 10^{-34} \mathrm{~J} \cdot \mathrm{s} .
$$

Meanwhile, the Planck constant has the following value [14]. 


$$
h=6.62607015 \times 10^{-34} \mathrm{~J} \cdot \mathrm{s} .
$$

$m_{\mathrm{e}} c \lambda_{\mathrm{C}}$ and $h$ are a perfect match. The currently known values for $m_{\mathrm{e}}$ or $\lambda_{\mathrm{C}}$ were not determined through experimentation. $m_{\mathrm{e}}$ was determined through precise calculations from Rydberg constant formulas, and $\lambda_{\mathrm{C}}$ was obtained by substituting $m_{\mathrm{e}}$ in the formula $\lambda_{\mathrm{C}}=h / m_{\mathrm{e}} c$.

Based on measured data from theoretical formulas or experiments designed to represent the fundamental laws of physics, many fundamental physical constants are being adjusted to avoid conflicts from arising between these constants. Because the formula to determine an electron's Compton wavelength is $\lambda_{\mathrm{C}}=h / m_{\mathrm{e}} c$, naturally the modified version of this Equation (17) is true. When we logically rewrite the Planck constant from Equation (11) into Equation (12) to include the frequency of a photon, we notice that non-frequency components $m_{\mathrm{e}} c \lambda$ are always constant, and this constant can be rewritten (defined) as $h$, a new constant.

While differing from historical development, we interpret (17) to mean not only that " $m_{\mathrm{e}} c \lambda_{\mathrm{C}}$ and $h$ are the same" but to mean that " $m_{\mathrm{e}} c \lambda_{\mathrm{C}}$ is defined as $h$ for sake of simplicity". Therefore, this paper does not claim the discovery of any relationship in Equation (17). However, because the Planck constant has historically been presumed to be a comparative constant used in Planck's harmonic oscillator energy quantum hypothesis and Einstein's light quantum hypothesis, it has taken on the image of being a discovered universal constant.

\section{Conclusions}

According to existing theory, Equation (11) and Equation (12) have been thought to have similar importance. However, according to our discussion, Equation (11) is the more fundamental of the two. Equation (12) is merely Equation (11) rewritten to also include frequency. The right side of Equation (19), the product of the physical quantities $m_{\mathrm{e}} c \lambda_{\mathrm{C}}$ except for frequency, is a steady value.

In this paper, we conclude that, theoretically, the Planck constant $h$ first comes into existence as a constant once we agree to name $m_{\mathrm{e}} c \lambda_{\mathrm{C}}$ as $h$. However, we have historically overlooked this agreement (definition). Then, the energy of a photon was presumed from the beginning to be proportional with its frequency; this proportional constant was named the Planck constant, and was considered to be a mysterious universal constant that ruled over the micro-world which could not be explained by classical mechanics.

There is no problem with introducing $h$ to simplify equations, but quantum mechanics is not affected even if there is no symbol $h$. Although it is bothersome, one can get by writing $m_{\mathrm{e}} c \lambda_{\mathrm{C}}$ instead of $h$. If one wishes to retain the Planck constant as a physical constant, then $h$ must be downgraded to an atomic or nuclear constant containing $m_{\mathrm{e}}$ and $\lambda_{\mathrm{C}}$.

However, the purpose of this paper is not to drag down the Planck constant from its position as a universal constant. I put together this paper to sound an alarm among physicists because the tendency to treat the Planck constant as sa- 
cred is too strong.

The physicists at the beginning of the 20th century overestimated the Planck constant, and this gave rise to universal constants that do not exist in the natural world in itself.

\section{Acknowledgements}

I would like to express my thanks to the staff at ACN Translation Services for their translation assistance.

\section{Conflicts of Interest}

The author declares no conflicts of interest regarding the publication of this paper.

\section{References}

[1] Planck, M. (1901) On the Law of Distribution of Energy in the Normal Spectrum. Annalen der Physik, 4, 553. https://doi.org/10.1002/andp.19013090310

[2] Bohr, N. (1952) Collected Works Vol 2. North-Holland, Amsterdam, 136.

[3] Suto, K. (2014) Previously Unknown Ultra-Low Energy Level of the Hydrogen Atom Whose Existence Can Be Predicted. Applied Physics Research, 6, 64-73. https://doi.org/10.5539/apr.v6n6p64

[4] Suto, K. (2020) Dark Matter and the Energy-momentum Relationship in a Hydrogen Atom. Journal of High Energy Physics, Gravitation and Cosmology, 6, 52-61. https://doi.org/10.4236/jhepgc.2020.61007

[5] Suto, K. (2014) $n=0$ Energy Level Present in the Hydrogen Atom. Applied Physics Research, 6, 109-115. https://doi.org/10.5539/apr.v6n5p109

[6] Suto, K. (2018) Derivation of a Relativistic Wave Equation more Profound than Dirac's Relativistic Wave Equation. Applied Physics Research, 10, 102-108. https://doi.org/10.5539/apr.v10n6p102

[7] Suto, K. (2019) The Relationship Enfolded in Bohr's Quantum Condition and a Previously Unknown Formula for Kinetic Energy. Applied Physics Research, 11, 19-34. https://doi.org/10.5539/apr.v11n1p19

[8] Suto, K. (2020) Relativistic Correction of the Rydberg Formula. Journal Modern Physics, 11, 294-303. https://doi.org/10.4236/jmp.2020.112018

[9] Suto, K. (2017) Presentation of Dark Matter Candidates. Applied Physics Research, 9, 70-76. https://doi.org/10.5539/apr.v9n1p70

[10] Suto, K. (2017) Region of Dark Matter Present in the Hydrogen Atom. Journal of Physical Mathematics, 8, Article ID: 1000252. https://doi.org/10.4172/2090-0902.1000252

[11] Suto, K. (2020) The Incompleteness of Quantum Mechanics Demonstrated by Considerations of Relativistic Kinetic Energy. Journal of Applied Mathematics and Physics, 8, 210-217. https://doi.org/10.4236/jamp.2020.82016

[12] Planck, M. (1901) On the Law of Distribution of Energy in the Normal Spectrum. Annalen der Physik, 4, 553. https://doi.org/10.1002/andp.19013090310

[13] Einstein, A. (1946) Elementary Derivation of the Equivalence of Mass and Energy. Technical Journal, 5, 16. 
[14] CODATA Internationally Recommended 2018 Values of the Fundamental Physical Constants. https://physics.nist.gov/cuu/Constants/index.html 